\title{
Is pancreatic exocrine insufficiency in celiac disease related to structural alterations in pancreatic parenchyma?
}

\author{
Surinder S. Ranaa, Arvind Dambalkara, Puneet Chhabraa, Ravi Sharmaa, Ritambhra Nadab, \\ Vishal Sharmaa, Satyavati Rana ${ }^{a}$, Deepak K. Bhasin ${ }^{a}$
}

Post Graduate Institute of Medical Education and Research, Chandigarh, India

Abstract

Background Although exocrine pancreatic insufficiency (EPI) has been reported in a number of patients with celiac disease (CD), it is not clear if this is primarily a functional or a structural defect. We studied pancreatic structural abnormalities by endoscopic ultrasound (EUS) in adult CD patients with EPI.

Methods Pancreatic exocrine function was prospectively assessed in 36 recently diagnosed CD patients (mean age: 29.8 years) by measuring fecal elastase. Pancreatic structural changes were assessed in CD patients with EPI by EUS and elastography. Exocrine functions were reassessed after 3 months of gluten-free diet.

Results Of the 36 CD patients included, 30 (83\%) had anemia, 21 (58\%) diarrhea, and 7 (19\%) hypothyroidism. Ten (28\%) patients had EPI with mean elastase levels of $141.6 \mu \mathrm{g} / \mathrm{g}$ of stool, of whom only one had a history of recurrent acute pancreatitis while the rest 9 patients had no history of acute or chronic pancreatitis. Of these 10 patients, $8(80 \%)$ had diarrhea, $8(80 \%)$ anemia, and 2 (20\%) hypothyroidism. EUS was done in 8 patients which showed: normal pancreas in 5 (50\%), hyperechoic strands in 3 (30\%), and hyperechoic foci without shadowing in 2 (20\%) patients. None had lobularity or parenchymal calcification. All patients except the patient with recurrent pancreatitis had normal strain ratio. Follow-up fecal elastase was within normal range in 6 of 7 (86\%) patients.

Conclusion EPI, assessed by fecal elastase levels in adult CD patients, possibly does not relate to structural alterations in the pancreatic parenchyma and may be reversible by following a gluten-free diet.

Keywords Celiac disease, pancreatitis, fecal elastase, endosonography, diarrhea

Ann Gastroenterol 2016; 29 (3): 1-4

\section{Introduction}

Celiac disease $(\mathrm{CD})$ is characterized by both intestinal and extraintestinalmanifestations. Exocrinepancreaticinsufficiency

Departments of ${ }^{\mathrm{a} G a s t r o e n t e r o l o g y}$ (Surinder S. Rana, Arvind Dambalkar, Puneet Chhabra, Ravi Sharma, Vishal Sharma, Satyavati

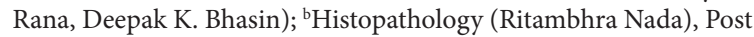
Graduate Institute of Medical Education and Research (PGIMER), Chandigarh, India

Conflict of Interest: None

Correspondence to: Dr Surinder S. Rana, MD, DM, Department of Gastroenterology, Post Graduate Institute of Medical Education and Research (PGIMER), Sector 12, Chandigarh 160012, India,

Tel.: +91 172 2756555, Fax: +91 172 27444401,

e-mail: drsurinderrana@yahoo.co.in

Presented at DDW 2015, Washington DC, USA

Received 16 March 2016; accepted 20 April 2016;

published online 11 May 2016

DOI: http://dx.doi.org/10.20524/aog.2016.0042
(EPI) has been reported in a number of patients with untreated CD [1-6]. The pathophysiology of EPI in CD is not fully understood and seems to be multifactorial. Impaired secretion of pancreatic stimulating hormones from diseased small intestine; deficiencies of amino acids because of malabsorption leading to reduction in pancreatic enzyme synthesis; and protein malnutrition causing structural changes of pancreas are some of the mechanisms proposed [6]. Case reports have also reported coexistence of pancreatitis with CD and a Swedish study has reported that patients with CD have an almost 3-fold increase in the risk of developing pancreatitis [7-9]. However, a recent study that used magnetic resonance imaging (MRI) to evaluate the pancreatic parenchymal structure did not find any morphological abnormalities in CD patients with EPI and thereby concluding that EPI is due to a functional defect in pancreatic enzyme secretion [10]. However, MRI can only detect advanced morphological changes of chronic pancreatitis (CP) while its ability to diagnose early and minimal CP is limited [11]. Since the ultrasound transducer can be placed in close proximity to the pancreas, endoscopic ultrasound (EUS) is now considered the most sensitive imaging modality for 
diagnosing pancreatic parenchymal changes [11]. Our aim was to assess the pancreatic structural abnormalities by EUS and EUS elastography in adult CD patients with EPI, diagnosed by impaired fecal elastase levels.

\section{Patients and methods}

This prospective study was conducted at a tertiary care center in North India. Thirty six consecutive adult recently diagnosed CD patients (mean age: $29.80 \pm 10.89$ years; 8 male) were prospectively enrolled. CD was diagnosed by serological testing using IgA anti-tissue transglutaminase antibody (IgAanti TTG) and histopathological examination of multiple small bowel biopsies. Histological changes were classified as per Marsh scoring system [12]. The study protocol was approved by our Institute's Ethics Committee and an informed consent was obtained from all the patients. Clinical, laboratory and demographic data were recorded for all CD patients recruited.

\section{Pancreatic exocrine function}

All patients underwent assessment for pancreatic exocrine function by measuring fecal elastase levels using an enzymelinked immunosorbent assay (ELISA). The liquid fecal samples were not considered for elastase measurement and repeat stool samples were collected in these patients. A commercially available kit (Bioserv Diagnostics, Germany) was used for measuring fecal elastase levels. Fecal elastase levels of $>200 \mu \mathrm{g} / \mathrm{g}$ of stool were considered as normal and values less than $200 \mu \mathrm{g} / \mathrm{g}$ of stool were considered as suggestive of EPI.

\section{Pancreatic endocrine function}

All patients were tested for endocrine insufficiency using glycosylated hemoglobin and fasting and postprandial blood sugar levels.

\section{Patients with low fecal elastase levels}

The pancreatic structural changes were evaluated in patients with EPI using EUS [11]. EUS was performed using a radial echoendoscope (EG-3670 URK radial echoendoscope, Pentax Inc., Tokyo, Japan) to look for both parenchymal and ductal changes of CP [11]. During the same procedure, EUS elastography was also performed as previously described $[11,13]$. In brief, for elastography, the EUS probe was in close proximity to the gastrointestinal (GI) wall and a stable image for at least $5 \mathrm{sec}$ was obtained. The region of interest (ROI) was manually selected to include the targeted area of the pancreas as well as surrounding tissue with two different areas (A and B) from the ROI being selected for quantitative analysis. Area A was the largest possible area of pancreas whereas area B was selected from normal surrounding GI wall. The quantitative result was defined as the quotient B/A-the strain ratio. The strain ratio was calculated at the head, body, and tail of the pancreas in each patient and the mean of these three values were taken as the final strain ratio. A strain ratio of $>2.25$ was used for diagnosing CP [13].

\section{Follow up}

All patients with EPI were started on a gluten-free diet and were followed up. No patient received pancreatic enzyme supplementation. Symptoms and body weight were reassessed quarterly. Fecal elastase levels were reassessed in patients who had been on a gluten-free diet for at least 3 months.

\section{Results}

Thirty-six adult recently diagnosed CD patients (mean age: $29.80 \pm 10.89$ years; 8 males) were prospectively studied. Anemia was the most common clinical presentation seen in $30(83 \%)$ patients and diarrhea was present in $21(58 \%)$ patients. Seven (19\%) patients had associated hypothyroidism. Duodenal histology revealed Marsh stage 0, 1, 2, 3A, 3B and $3 \mathrm{C}$ in 0 (0\%), 5 (14\%), 5 (14\%), 0 (0\%), 16 (44\%), and $10(28 \%)$ patients, respectively. EPI, indicated by the low fecal elastase, was diagnosed in $10(28 \%)$ patients (mean age: $30.4 \pm 8.8$ years; 8 female).

\section{Patients with low fecal elastase $(n=10)$}

Of the 10 patients with low fecal elastase, 8 (80\%) had diarrhea, 8 (80\%) anemia, and 2 (20\%) hypothyroidism (Table 1). Duodenal histology revealed Marsh stage 2, 3B and $3 \mathrm{C}$ in $1(10 \%), 4(40 \%)$, and 5 (50\%) patients, respectively. One of these 10 patients had a history of recurrent acute pancreatitis, while the rest 9 patients had no history suggestive of acute or CP. Mean fecal elastase levels were $141.60 \pm 19.32 \mu \mathrm{g} / \mathrm{g}$ of stool. Nine $(90 \%)$ patients with low fecal elastase had villous atrophy compared to 16 (65\%) patients with normal fecal elastase $(\mathrm{P}=0.2)$. All patients had normal glycosylated hemoglobin as well as fasting and postprandial blood sugar levels.

\section{EUS and EUS elastography findings}

EUS was done in 8 (80\%) patients with low fecal elastase as 2 patients refused consent. EUS revealed normal pancreas in 5 patients, hyperechoic strands in 3, and hyperechoic foci without shadowing in 2 patients (Figs. 1 and 2). None of the patients had lobularity or parenchymal calcification on EUS. The main pancreatic duct had a normal appearance in all of these 8 patients. EUS elastography revealed a normal strain 
Table 1 Demographic, clinical and investigational profile of ten patients with low fecal elastase

\begin{tabular}{|c|c|c|c|c|c|c|c|c|c|}
\hline Sr. No & Age (yrs) & Sex & Diarrhea & Hypothyroid & Anemia & Marsh staging & Strain ratio & Fecal elastase $(\mu \mathrm{g} / \mathrm{g})$ & History of pancreatitis \\
\hline 1 & 41 & $\mathrm{~F}$ & No & Yes & No & 2 & 1.6 & 133 & No \\
\hline 2 & 29 & $\mathrm{~F}$ & Yes & No & Yes & $3 \mathrm{C}$ & 1.4 & 140 & No \\
\hline 3 & 21 & M & Yes & No & No & $3 \mathrm{~B}$ & 1.9 & 140 & No \\
\hline 4 & 32 & $\mathrm{~F}$ & Yes & No & Yes & $3 \mathrm{~B}$ & - & 173 & No \\
\hline 5 & 23 & $\mathrm{~F}$ & Yes & No & Yes & $3 \mathrm{C}$ & 2.1 & 140 & No \\
\hline 6 & 27 & $\mathrm{~F}$ & Yes & Yes & Yes & $3 \mathrm{C}$ & 1.7 & 170 & No \\
\hline 7 & 38 & $\mathrm{~F}$ & Yes & No & Yes & $3 \mathrm{C}$ & - & 115 & No \\
\hline 8 & 19 & M & Yes & No & Yes & $3 \mathrm{~B}$ & 1.6 & 150 & No \\
\hline 9 & 28 & $\mathrm{~F}$ & No & No & Yes & $3 \mathrm{~B}$ & 2.1 & 115 & No \\
\hline 10 & 46 & $\mathrm{~F}$ & Yes & No & Yes & $3 \mathrm{C}$ & 3.6 & 140 & Yes (RAP) \\
\hline
\end{tabular}

$M$, male; F, female; RAP, recurrent acute pancreatitis

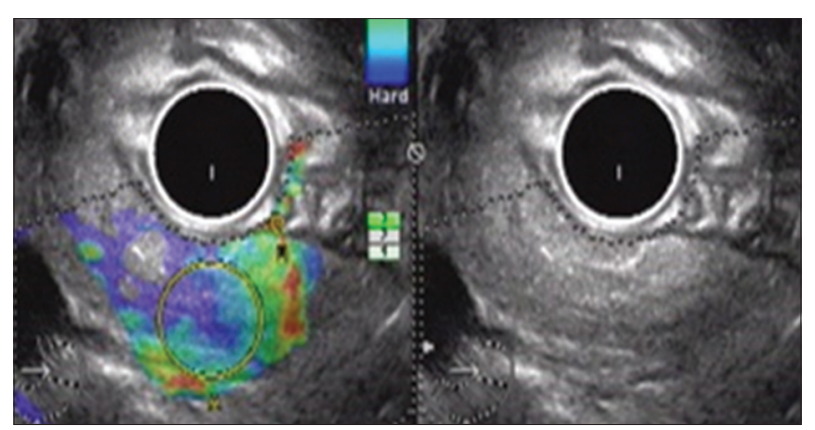

Figure 1 Endoscopic ultrasound elastography in a patient with recurrent pancreatitis: hyperechoic foci and strands with a strain ratio of 3.62

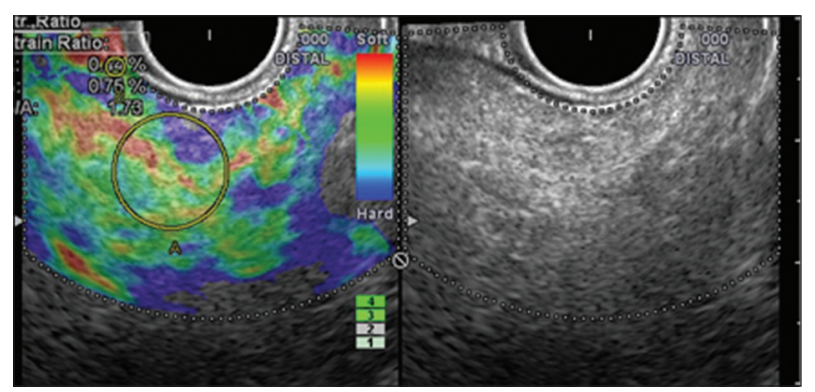

Figure 2 Endoscopic ultrasound elastography in a patient with no pancreatic symptoms: hyperechoic foci and strands with a strain ratio of 1.73

ratio in all patients except of one patient who had recurrent acute pancreatitis. The mean strain ratio in these seven patients with normal EUS elastography was $1.77 \pm 0.26$. The patient who had recurrent acute pancreatitis had a strain ratio of 3.62 and hyperechoic strands and foci on EUS.

\section{Follow up after starting gluten-free diet}

Patients with EPI were started on a gluten-free diet and followed up regularly for clinical response. All patients had clinical response on the gluten-free diet. No patient required pancreatic enzyme supplementation. Fecal elastase levels were reassessed in 7 patients after a median follow up of 6 months. Fecal elastase levels normalized in $6(86 \%)$ patients. The patient with the low fecal elastase levels (i.e. the patient with recurrent pancreatitis) had a marked clinical response with complete remission of diarrhea after the onset of gluten-free diet alone.

\section{Discussion}

Our study has confirmed the presence of EPI in one third of the untreated CD patients. The reported frequency of EPI measured by various non invasive methods ranged between $11.4 \%$ and $56.2 \%$, with the higher frequency reported in $\mathrm{CD}$ patients having diarrhea in spite of being on gluten-free diet $[1,5]$. We also found that the frequency of diarrhea was similar in patients with or without EPI ( $80 \%$ vs. $50 \%$; $\mathrm{P}=0.5)$. However, the response to gluten-free diet and the higher frequency of villous atrophy in patients with EPI suggests that EPI plays a minor etiological role in diarrhea in these patients. This observation is in accordance with previously published reports showing that the majority of patients with EPI respond to gluten-free diet and have enzyme values above the threshold value considered to cause malabsorption and diarrhea [1-6].

The pathophysiology of EPI in CD is not fully understood. Impaired secretion of pancreatic stimulating hormones from the atrophied proximal small intestine is considered to be the most likely mechanism for EPI. This hypothesis is supported by immunohistochemical studies that have shown significant alterations in enteric endocrine cells with significant decrease in secretin cells [14]. However, some studies have shown an increased frequency of pancreatitis in CD patients as well as persistence of diarrhea and EPI in spite of being on gluten-free diet [5,7]. These studies raise the question whether structural changes in pancreas contribute to EPI in CD patients. 
We attempted to answer this question by using EUS and EUS elastography and found that all but one patient with EPI had normal pancreas. EUS features of CP include both parenchymal and ductal changes [11]. The Rosemont criteria developed by leading experts has defined precisely each EUS criterion and include major and minor criteria $[11,15]$. The major criteria include echogenic structures $\geq 2 \mathrm{~mm}$ in length/width with shadowing, parenchymal lobularity with honeycombing and echogenic structures within the main pancreatic duct with acoustic shadowing suggestive of ductal calculi. None of our patients had these major EUS features of CP.

In addition, normalization of fecal elastase levels in $86 \%$ of patients after $>3$ months of gluten-free diet suggests that EPI, assessed by fecal elastase levels in adult CD patients, is not due to structural alterations in the pancreatic parenchyma. A similar observation has been reported by a recent study by Vujasinovic et al where the authors used MRI to assess the structural changes of pancreas in CD patients with EPI, assessed by fecal elastase [10]. In this study, the authors found EPI in 4 (4.4\%) patients, and MRI was normal in 3 patients. In one patient with diabetes, MRI showed an atrophic pancreas but in all four patients the signal intensity was normal with no signs of fatty infiltration and no dilatation of pancreatic duct or any of the side branches.

Use of EUS elastography to exclude structural changes in pancreas in CD patients with EPI is the most important strength of this study. However, our study has a number of

\section{Summary Box}

\section{What is already known:}

- Celiac disease (CD) is characterized by both intestinal and extra intestinal manifestations

- Exocrine pancreatic insufficiency (EPI) has been reported in a number of untreated CD patients

- The pathophysiology of EPI in CD is not fully understood and seems to be multifactorial

- It is not clear if this is primarily a functional or a structural defect of the pancreas

\section{What the new findings are:}

- EPI was seen in one third of untreated CD patients

- EPI, assessed by fecal elastase levels in adult CD patients, is possibly not related to structural alterations in the pancreatic parenchyma, since the majority of these patients had normal endoscopic ultrasound elastography findings

- EPI may be reversible by gluten-free diet in the majority of patients limitations. The study sample was small and was conducted at a single center. Furthermore, utility of fecal elastase for diagnosing EPI has some limitations, such as a poor sensitivity to diagnose mild-to-moderate EPI [16].

In conclusion, EPI, assessed by fecal elastase levels in adult $\mathrm{CD}$ patients, is possibly not related to structural alterations in the pancreatic parenchyma and may be reversible by glutenfree diet in the majority of the patients.

\section{References}

1. Pezzilli R. Exocrine pancreas involvement in celiac disease: a review. Recent Pat Inflamm Allergy Drug Discov 2014;8:167-172.

2. Evans KE, Leeds JS, Morley S, Sanders DS. Pancreatic insufficiency in adult celiac disease: do patients require long-term enzyme supplementation? Dig Dis Sci 2010;55:2999-3004.

3. Carroccio A, Iacono G, Montalto G, et al. Exocrine pancreatic function in children with coeliac disease before and after a gluten free diet. Gut 1991;32:796-799.

4. Weizman Z, Hamilton JR, Kopelman HR, et al. Treatment failure in coeliac disease due to coexistent exocrine pancreatic insufficiency. Pediatrics 1987;80:924-926.

5. Leeds JS, Hopper AD, Hurlstone DP, et al. Is exocrine pancreatic insufficiency in adult coeliac disease a cause of persisting symptoms? Aliment Pharmacol Ther 2007;25:265-271.

6. Freeman HJ. Pancreatic endocrine and exocrine changes in celiac disease. World J Gastroenterol 2007;13:6344-6346.

7. Sadr-Azodi O, Sanders DS, Murray JA, Ludvigsson JF. Patients with celiac disease have an increased risk for pancreatitis. Clin Gastroenterol Hepatol 2012;10:1136-1142.e3.

8. Rana SS, Bhasin DK, Sinha SK, Singh K. Coexistence of chronic calcific pancreatitis and celiac disease. Indian J Gastroenterol 2007;26:150.

9. Arya S, Rana SS, Sinha SK, Nagi B, Bhasin DK. Celiac disease and chronic calcific pancreatitis with pancreas divisum. Gastrointest Endosc 2006;63:1080-1081.

10. Vujasinovic M, Tepes B, Volfand J, Rudolf S. Exocrine pancreatic insufficiency, MRI of the pancreas and serum nutritional markers in patients with coeliac disease. Postgrad Med J 2015;91:497-500.

11. Rana SS, Vilmann P. Endoscopic ultrasound features of chronic pancreatitis: A pictorial review. Endosc Ultrasound 2015;4:10-14.

12. Marsh MN. Gluten, major histocompatibility complex, and the small intestine. A molecular and immunobiologic approach to the spectrum of gluten sensitivity ('celiac sprue'). Gastroenterology 1992;102:330-354.

13. Iglesias-Garcia J, Domínguez-Muñoz JE, Castiñeira-Alvariño M, Luaces-Regueira M, Lariño-Noia J. Quantitative elastography associated with endoscopic ultrasound for the diagnosis of chronic pancreatitis. Endoscopy 2013;45:781-788.

14. Buchan AM, Grant S, Brown JC, Freeman HJ. A quantitative study of enteric endocrine cells in celiac sprue. J Pediatr Gastroenterol Nutr 1984;3:665-671.

15. Stevens T, Lopez R, Adler DG, et al. Multicenter comparison of the interobserver agreement of standard EUS scoring and Rosemont classification scoring for diagnosis of chronic pancreatitis. Gastrointest Endosc 2010;71:519-526.

16. Loser C, Mollgaard A, Folsch U. Fecal elastase 1: A novel, highly sensitive, specific tubeless pancreatic function test. Gut 1996;39:580-586. 